\title{
Water repellency improvement of cellulosic textile fibers by betulin and a betulin-based copolymer
}

\author{
Tianxiao Huang $\mathbb{D} \cdot$ Dongfang $\mathrm{Li} \cdot$ Monica Ek $\mathbb{E}$
}

Received: 2 November 2017/ Accepted: 1 February 2018/Published online: 8 February 2018

(C) The Author(s) 2018. This article is an open access publication

\begin{abstract}
Betulin is a naturally abundant and hydrophobic compound in the outer bark of birch and can readily be obtained by solvent extraction. Here, solutions of betulin were used to treat cotton fabrics and improve their water repellency. Cotton fabric impregnated in a solution of betulin in ethanol showed a contact angle for water of approximately $153^{\circ}$ and reached a water repellency score of 70 according to a standard water repellency test method. A betulin-terephthaloyl chloride (TPC) copolymer was synthesized. Both betulin and betulin-TPC copolymer were characterized by nuclear magnetic resonance spectroscopy and Fourier transform infrared spectroscopy. The copolymer was characterized by size exclusion chromatography and differential scanning calorimetry. When impregnated with a solution of betulin-TPC copolymer in tetrahydrofuran, a cotton fabric showed a water contact angle of $151^{\circ}$ and also reached a water repellency score of 70 . Films based on betulin and betulin-TPC copolymer were prepared and coated onto the surface of the fabrics by compression molding. These coated fabrics showed water contact angles of $123^{\circ}$ and $104^{\circ}$ respectively and each reached a water repellency score of 80 .
\end{abstract}

T. Huang · D. Li · M. Ek $(\bowtie)$

Department of Fiber and Polymer Technology, School of

Engineering Sciences in Chemistry, Biotechnology and

Health, KTH Royal Institute of Technology,

10044 Stockholm, Sweden

e-mail: monicaek@kth.se
Keywords Betulin - Cellulose - Contact angle · Copolymer $\cdot$ Textile $\cdot$ Water repellency

\section{Introduction}

Cellulosic textile fibers such as cotton have advantages over synthetic fibers due to their economic convenience, softness and sustainability, but cotton fabric can easily be wetted by water because abundance of hydroxyl groups within the cellulose molecules (Xu and Cai 2008). For cases when water repellency is important, this property is not desired (Bae et al. 2009). Water repellent fabrics are needed in e.g., rainwear, self-cleaning coatings, anti-fouling or anti-corrosion coatings, and bandages (Abidi and Hequet 2004; Sahin 1996; Shi et al. 2013; Singh 1987). Therefore, the hydrophobization of cellulose fibers has recently attracted considerable attentions (Bae et al. 2009; Ivanova and Zaretskaya 2010; Shi et al. 2013; Wi et al. 2009).

Over the past two centuries, scientists have made plenty of effort to explore the mechanism of wetting. A series of wetting models with related equations have been proposed (Cassie and Baxter 1944; Wenzel 1936; Young 1805). In general, there are two ways to increase the water repellency of textiles: by lowering the surface energy and by creating a hierarchical structure to increase the surface roughness (Shi et al. 
2013). The first way is to introduce low surface energy substances such as a paraffin wax or paraffin emulsion onto the surface of the textile. This method is easy but comes some problems such as lack of air and vapor permeability, as well as low comfort (Hagenmaier and Shaw 1991), and thus this method is not widely used nowadays. The alternative way is relatively complex but does not affect the comfort of the textiles. In order to build up a hierarchical structure and increase the surface roughness, nano/micro-scale texture is first created on the surface of the textile fiber by introducing some nano-scale particles like $\mathrm{SiO}_{2}, \mathrm{TiO}_{2}$ and $\mathrm{ZnO}$ (Bae et al. 2009). Afterwards, some waxy materials like fluorinated silanes are added to make the textile waterproof (Shi et al. 2013). Unfortunately, the fluorinated silanes are expensive and contaminated wastewater is generated during the treatment process (Lee et al. 2005; Mazurek et al. 2001; Yahaya et al. 2001).

The current common solutions are thus neither economic nor environmentally friendly, which motivates a study to find an environmentally friendly way or substance to make the cellulosic textile fibers water repellent. Substances isolated from natural resources may be a good choice. Betulin, a naturally abundant compound in the outer bark of birch, can be readily obtained by solvent extraction (Fridén et al. 2016). Betulin represents $25-35 \%$ of the weight of the outer bark of major birch species (Krasutsky 2006), and due to its hydrocarbon skeleton, betulin possesses very low surface energy. This characteristic suggests that betulin should be exploited for hydrophobization treatment instead of simply being burnt together with the bark to provide thermal energy. In fact, the kraft digestion process in the paper industry cannot fully dissolve the betulin accompanying the incompletely debarked wood, and hence the undesired betulin can cause deposition problems in the manufacture process (Sharoyan 2011). Thus, using this side-stream product for value-added applications is one aim of this study, where betulin and a copolymer developed from betulin have been exploited to improve the water repellency of cotton fabrics.

In this study, betulin was extracted from the outer bark of birch and a copolymer was synthesized from betulin and terephthaloyl chloride (TPC). Both betulin and the betulin-TPC copolymer were characterized by nuclear magnetic resonance spectroscopy (NMR) and Fourier transform infrared spectroscopy (FTIR), and the copolymer was also characterized by size exclusion chromatography (SEC) and differential scanning calorimetry (DSC). Two surface treatment techniques were applied: impregnation of fabric samples in solutions followed by drying; preparation of films followed by compression molding. All the surfacetreated fabrics were characterized by water contact angle measurements, field-emission scanning electron microscopy (SEM) imaging and a standard water repellency test method.

\section{Experimental}

\section{Materials}

The outer bark of birch (Betula verrucosa) was collected in the park next to KTH Royal Institute of Technology, in Stockholm, Sweden, and then dried in air at ambient temperature. The dried bark was ground to 40 mesh particles, and Soxhlet extracted for $48 \mathrm{~h}$ with n-heptane as solvent. Betulin was obtained as a white powder after solvent evaporation and drying. The purity of the betulin was $80 \%$, as determined by ${ }^{1} \mathrm{H}$ NMR. The remaining $20 \%$ may consist mainly of lupeol and betulinic acid (Krasutsky 2006). The chemical structures of betulin, lupeol, and betulinic acid are shown in scheme 1.

${ }^{1} \mathrm{H}$ NMR $\left(\mathrm{CDCl}_{3}\right) \delta(\mathrm{ppm}): 4.70(\mathrm{~s}, 1 \mathrm{H}, 29 \mathrm{a}-\mathrm{H})$, 4.60 (s, 1H, 29b-H), 3.83 (d, 1H, 28b-H), 3.36 (d, 1H, 28a-H), $3.21(\mathrm{dd}, 1 \mathrm{H}, 3-\mathrm{H}), 2.41(\mathrm{~m}, 1 \mathrm{H}, 19-\mathrm{H}), 1.70$ (m, 3H, 30-H), 1.67-0.70 (m, 40H).

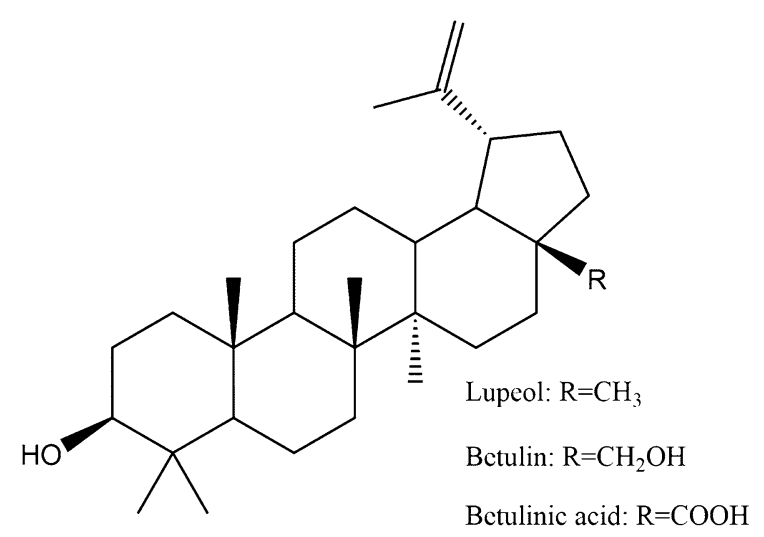

Scheme 1 Chemical structure of lupeol, betulin and betulinic acid 
Commercial $100 \%$ cotton table runners were purchased from Hemtex, Stockholm, Sweden. A green dye produced by Dr. Oetker Sverige AB was purchased from T-Snabben, Stockholm, Sweden. The following chemicals were used as received without further purification: Chloroform (99.9\%), terephthaloyl chloride ( $\geq 99 \%$ ), tetrahydrofuran (THF, $99.99 \%)$, sodium hydroxide $(\mathrm{NaOH})$, and $1 \mathrm{M}$ hydrochloric acid (HCl) (Sigma-Aldrich); ethyl acetate $(99.98 \%)$ and pyridine (> 99\%) (Fisher Scientific); ethanol (96\%) (VWR International).

Pretreatment of cotton fabric samples

The cotton table runner was cut into $18 \mathrm{~cm} \times 18 \mathrm{~cm}$ pieces, and washed in a laundering machine at $40{ }^{\circ} \mathrm{C}$ for $2 \mathrm{~h}$ with non-bleaching washing powder to remove greasy dirt. Afterwards, the fabric samples were further washed with a $2 \mathrm{~g} \mathrm{~L}^{-1} \mathrm{NaOH}$ solution for $90 \mathrm{~min}$ at $98^{\circ} \mathrm{C}$. Finally, the samples were washed in distilled water and dried at $105{ }^{\circ} \mathrm{C}$ overnight (Wang et al. 2006).

Preparation of a betulin-terephthaloyl chloride copolymer

The betulin-TPC copolymer was prepared as previously described (Jeromenok 2012). Briefly: a mixture of betulin and TPC in a molar ratio of 1:3 was dissolved in toluene together with an excess of pyridine. Molecular sieves were added to the solution. The reaction was carried out at $105{ }^{\circ} \mathrm{C}$ for $24 \mathrm{~h}$, and terminated by cooling to ambient temperature. After solvent evaporation, the crude product was washed with $1 \mathrm{M} \mathrm{HCl}$ solution and recrystallized in ethanol. The betulin-TPC copolymer was obtained as a yellowish powder after drying at $60{ }^{\circ} \mathrm{C}$ overnight, and the structure was characterized by ${ }^{1} \mathrm{H}$ NMR. ${ }^{1} \mathrm{H}$ NMR $\left(\mathrm{CDCl}_{3}\right) \delta(\mathrm{ppm}): 8.13\left(\mathrm{~s}, 4 \mathrm{H}, \mathrm{C}_{\mathrm{ar}}-\mathrm{H}\right), 4.75(\mathrm{~s}$, 1H, 29a-H), 4.65 (s, 1H, 29b-H), 4.58 (d, 1H, 28b-H), 4.15 (d, 1H, 28a-H), 3.76 (dd, 1H, 3-H), 2.55 (s, 1H, 19-H), 1.88-0.94 (m, 42H, $\left.\mathrm{C}_{\mathrm{sp} 3}-\mathrm{H}\right)$.

Film preparation

As shown in Table 1, cellulose triacetate (CTA) was mixed with betulin or betulin-TPC copolymer to prepare mixtures, and each mixture was dissolved in $6 \mathrm{~mL}$ of chloroform. Films 1-11 were obtained after solvent casting. A pure CTA film was prepared using the same procedure and used as reference.

Impregnation of cotton fabric samples

with solutions (methods A and B)

The fabric samples were impregnated in solutions of betulin (3.75 and $7.5 \mathrm{~g} \mathrm{~L}^{-1}$ in ethanol, $7.5 \mathrm{~g} \mathrm{~L}^{-1}$ in ethyl acetate) and betulin-TPC copolymer (3.75 $\mathrm{g} \mathrm{L}^{-1}$ in THF) to prepare materials A1, A2, A3 and B respectively, as shown in Fig. 1.

Surface coating of cotton fabric samples with films (methods C and D)

The films were surface coated on fabric samples by a Fontijne TPB compress molding instrument under a pressure of $80 \mathrm{kN}$ at $210{ }^{\circ} \mathrm{C}$ for $4 \mathrm{~min}$ to obtain materials $\mathrm{C}$ and $\mathrm{D}$ respectively, as shown in Fig. 1.

\section{Characterization}

${ }^{1} \mathrm{H}$ Nuclear magnetic resonance (NMR) spectra of betulin and of the betulin-TPC copolymer were recorded on a Bruker UltraShield $400 \mathrm{MHz}$ spectrometer (Germany). Deuterated chloroform $\left(\mathrm{CDCl}_{3}\right)$ was used as the solvent.

Size exclusion chromatography (SEC) was performed on a system consists of a Viscotek TDA model 301 system equipped with two LT4000L columns $(7.8 \times 300 \mathrm{~mm})$, a VE $2500 \mathrm{GPC}$ autosampler, a VE 1121 GPC pump, a VE 3580 RI detector, and a VE 5710 GPC degasser (UK). The eluent was THF. The betulin-TPC copolymer was dissolved in THF to give a solution with a concentration of $2 \mathrm{~g} \mathrm{~L}^{-1}$. Prior to injection, the solution was filtered through a PTFE syringe filter. Calibration was achieved using narrow linear polystyrene standards with molecular weights ranging from 1200 to 400,000 Da.

Fourier transform infrared spectroscopy (FTIR) spectra (absorbance mode) of betulin and betulin-TPC copolymer were recorded on a Perkin-Elmer Spectrum 2000 FT-IR equipped with a heat-controlled singlereflection attenuated total reflection accessory (Golden Gate heat-controlled); Each spectrum was recorded between 4000 and $600 \mathrm{~cm}^{-1}$ with 32 scans.

Differential scanning calorimetry (DSC) was performed on a Mettler Toledo DSC 820 calorimeter equipped with a sample robot and a cryocooler. The 
Table 1 Cellulose triacetate (CTA) mixed with betulin or betulin-TPC copolymer to cast films

${ }^{\text {a}}$ Mass ratio of CTA to Betulin or betulin-TPC copolymer

Fig. 1 Schematic illustration of experimental methods. Method A: Fabric impregnated in betulin solutions with ethanol (3.75 and $\left.7.5 \mathrm{~g} \mathrm{~L}^{-1}\right)$ or ethyl acetate $\left(7.5 \mathrm{~g} \mathrm{~L}^{-1}\right)$ as solvent, affording materials A1, 2 and 3 respectively; Method B: Fabric impregnated in betulin-TPC copolymer solution (3.75 $\left.\mathrm{g} \mathrm{L}^{-1}\right)$, affording material B; Methods C and D: Fabrics coated by Betulin or Betulin-TPC copolymer based films, affording materials $\mathrm{C}$ and $\mathrm{D}$ respectively

\begin{tabular}{lllll}
\hline Mixture/film & CTA $(\mathrm{mg})$ & Betulin $(\mathrm{mg})$ & Copolymer $(\mathrm{mg})$ & Ratio $^{\mathrm{a}}$ \\
\hline 1 & 290 & 116 & - & $1: 0.4$ \\
2 & 290 & 203 & - & $1: 0.7$ \\
3 & 290 & 290 & - & $1: 1$ \\
4 & 290 & 377 & - & $1: 1.3$ \\
5 & 290 & 461 & - & $1: 1.6$ \\
6 & 290 & 551 & - & $1: 1.9$ \\
7 & 290 & - & 116 & $1: 0.4$ \\
8 & 290 & - & 203 & $1: 0.7$ \\
9 & 290 & - & 290 & $1: 1$ \\
10 & 290 & - & 377 & $1: 1.3$ \\
11 & 290 & - & 461 & $1: 1.6$ \\
\hline
\end{tabular}
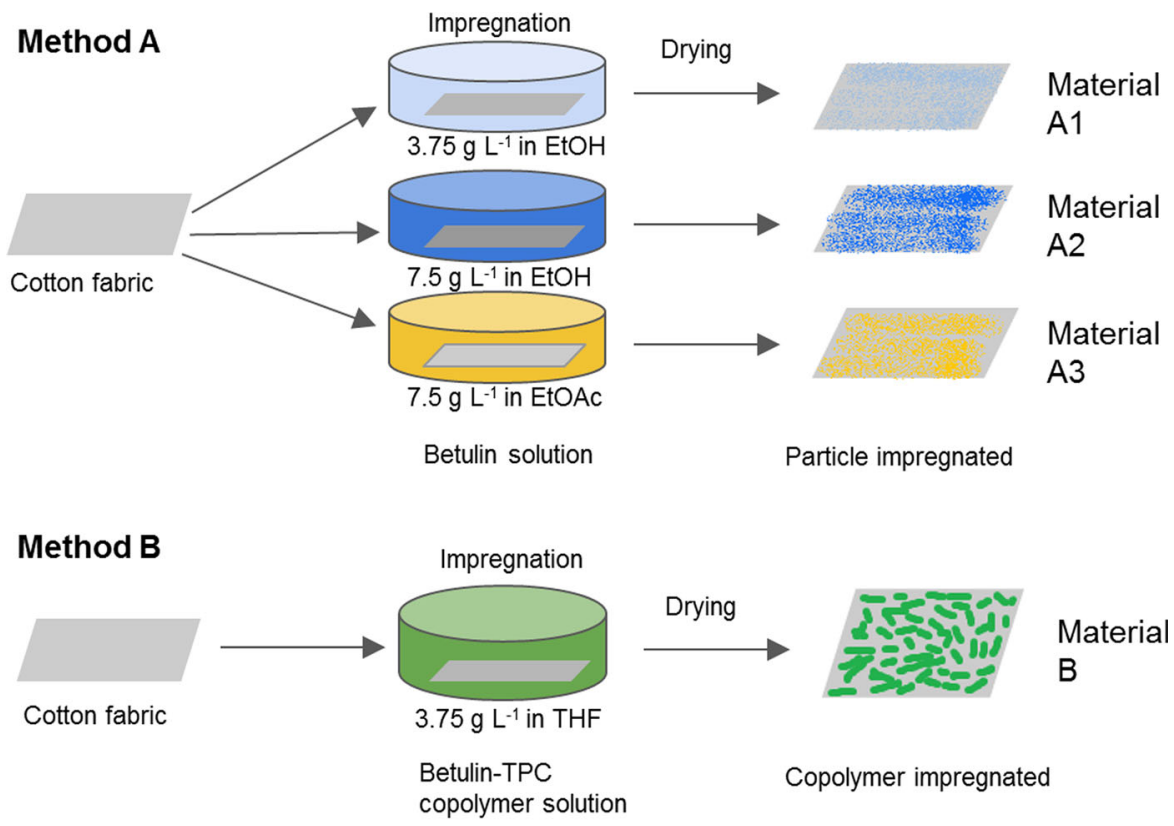

\section{Method C}

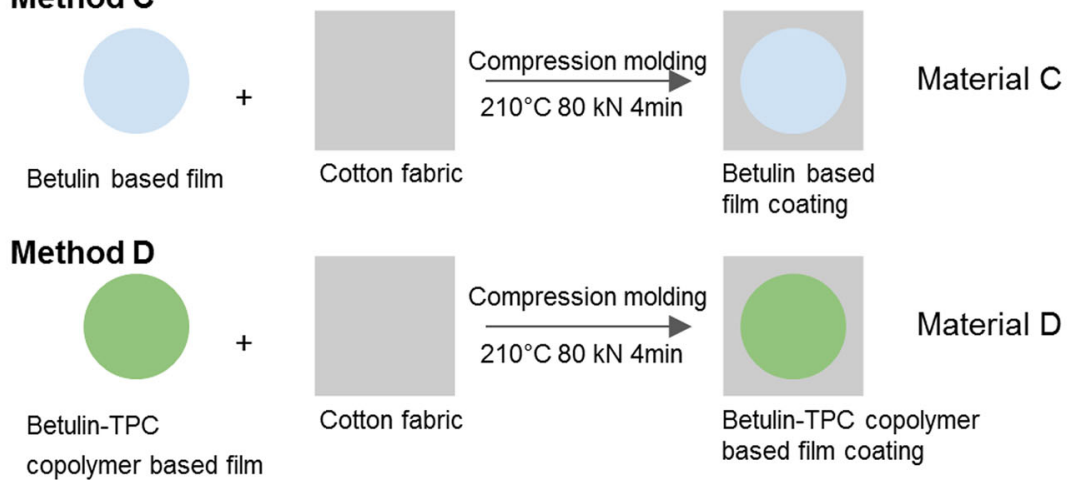

process was conducted in a $\mathrm{N}_{2}$ atmosphere with a gas flow of $50 \mathrm{~mL} \mathrm{~min}^{-1}$. Approximately $5 \mathrm{mg}$ of
Betulin-TPC copolymer sample was placed in a $40-\mu \mathrm{L}$ aluminum pan and heated from 25 to $300{ }^{\circ} \mathrm{C}$ 
$\left(5{ }^{\circ} \mathrm{C} \mathrm{min}{ }^{-1}\right)$, cooled from 300 to $-30{ }^{\circ} \mathrm{C}\left(-5{ }^{\circ} \mathrm{C}\right.$ $\left.\min ^{-1}\right)$, and then heated to $300{ }^{\circ} \mathrm{C}\left(5^{\circ} \mathrm{C} \min ^{-1}\right)$.

Scanning electron microscopy (SEM) images were captured by a Hitachi S-4800 Field-emission scanning electron microscopy. After adhesion onto a round metal stud, the samples were coated with a $4 \mathrm{~nm}$ gold layer by a Cressington 208HR high-resolution sputter coater, which was connected with a Cressington thickness monitor controller.

Water contact angle (WCA) was measured on a KSV Instruments CAM 200-m connected with a Basler A602f camera. A measurement was made at 5 different positions on each sample. A $10 \mu \mathrm{L}$ water droplet was placed on the surface and pictures were taken every $10 \mathrm{~s}$ for a total period of $170 \mathrm{~s}$. The contact angle for each sample was averaged over 5 positions.

The American Association of Textile Chemists and Colorists (AATCC) water repellency test was employed: $250 \mathrm{~mL}$ of water was sprayed onto a taut surface of each material using a specific tester. After spraying, the wet pattern of the material was compared with the patterns in a standard rating chart to rate the material's water repellency (AATCC TM22-2014, water repellency: spray test).

\section{Result and discussion}

Preparation of a betulin-terephthaloyl chloride copolymer

Several syntheses were performed under different conditions to determine the best reaction conditions, and the method was selected where $0.15 \mathrm{~mol} \mathrm{~L}^{-1}$ of betulin solution in toluene (molar ratio of betulin to TPC 1:3) was reacted at $105{ }^{\circ} \mathrm{C}$ for $24 \mathrm{~h}$ with excess pyridine as catalyst to give a final product with a relative large molecular weight and low PDI. The

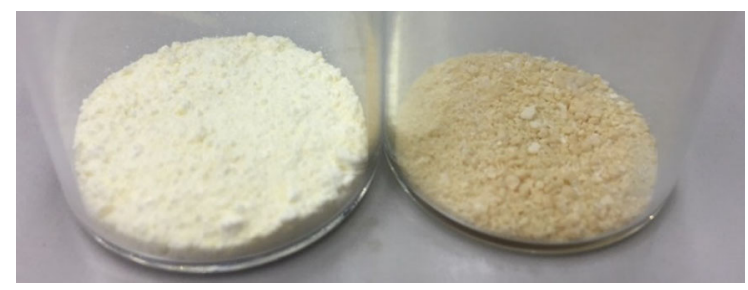

Fig. 2 Betulin (left) and betulin-TPC copolymer (right) appearance of betulin and synthesized copolymer are shown in Fig. 2.

As shown in Scheme 2, the pyridine acts as both nucleophilic catalyst and acid-binding reagent. Pyridine is a stronger nucleophile than betulin (Vasnev et al. 1987) and thus attacks TPC before the betulin to generate a positively charged electrophilic intermediate which then reacts with betulin to give the ester. Furthermore, pyridine is a Lewis base, which consumes the generated hydrochloric acid to move the reaction equilibrium to the right. The $\mathrm{Mw}$ or $\mathrm{Mn}$ of the copolymer synthesized in this study was generally lower than that of copolymers synthesized in previous studies (Jeromenok 2012). A hypothesis is that impurities with only one hydroxyl, such as lupeol (Krasutsky 2006), stop the propagation of the copolymer chain as soon as it is involved in the reaction.

According to SEC, the bulk synthesized betulinTPC copolymer had a $\mathrm{M}_{\mathrm{w}}$ of $9000 \mathrm{Da}, \mathrm{M}_{\mathrm{n}}$ of $2890 \mathrm{Da}$, and a PDI of 3.1. The DSC curve (Fig. 3) shows that the glass transition temperature $\left(\mathrm{T}_{\mathrm{g}}\right)$ of the amorphous region in the betulin-TPC copolymer was approximately $253{ }^{\circ} \mathrm{C}$. No other signal was observed within the range of detection.

Betulin and betulin-TPC copolymer were analyzed by FTIR, as shown in Fig. 4. The spectra of betulin and copolymer show bands at $1645 \mathrm{~cm}^{-1}$, corresponding to the stretching vibration of $\mathrm{C}=\mathrm{CH}_{2}$ in isopropenyl group, indicating that the isopropenyl group is present in both the betulin and copolymer structures, the two spectra were thus normalized with respect to this peak. The spectrum of betulin-TPC copolymer shows strong bands at $1716 \mathrm{~cm}^{-1}$, corresponding to the stretching of carbonyl groups $(\mathrm{C}=\mathrm{O})$, and bands at 1264 and $1100 \mathrm{~cm}^{-1}$, corresponding to the asymmetric and symmetric stretching of C-O bonds within ester bonds respectively, indicating the formation of ester bonds as a result of the polycondensation. It should be noted that the absorption band of $\mathrm{C}=\mathrm{O}$ stretching appears at $1716 \mathrm{~cm}^{-1}$ in betulin-TPC copolymer was lower than the range of $1735-1750 \mathrm{~cm}^{-1}$ where the $\mathrm{C}=\mathrm{O}$ (ester) band normally appears. An explanation for the lower wavenumber of $\mathrm{C}=\mathrm{O}$ groups is that the conjugative effect of the $\mathrm{C}=\mathrm{O}$ groups and their connected aromatic rings in betulin-TPC copolymer weakened the stretching vibration of the $\mathrm{C}=\mathrm{O}$ groups.

The spectrum of betulin shows a broad band from 3650 to $3200 \mathrm{~cm}^{-1}$, corresponding to the hydrogenbonded hydroxyls. Compared with the spectrum of 


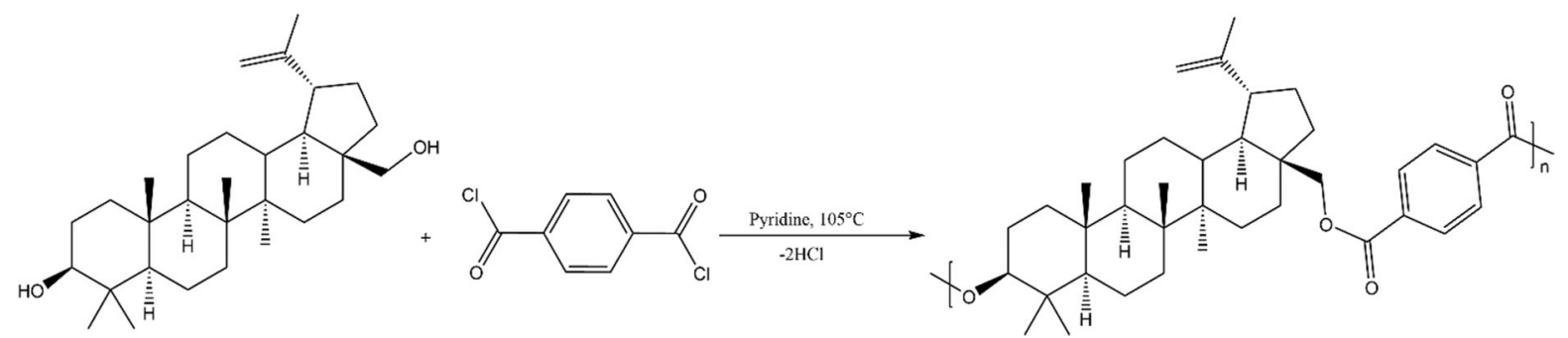

Scheme 2 Synthesis of betulin-TPC copolymer at $105{ }^{\circ} \mathrm{C}$ with pyridine as a catalyst. Reproduced with permission from Jeromenok (2012)

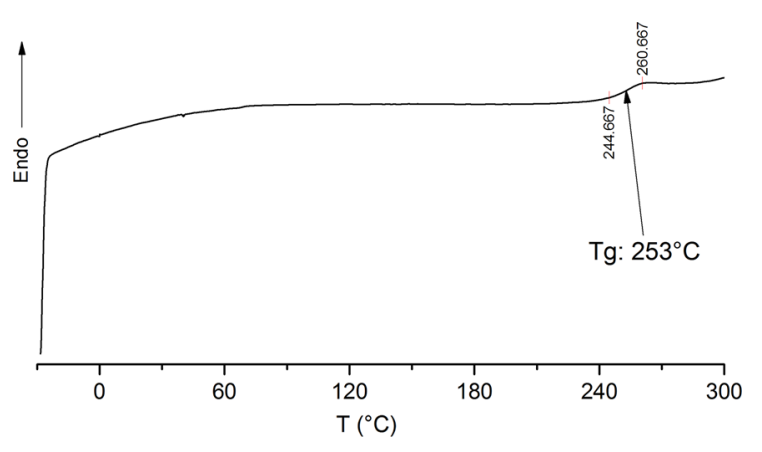

Fig. 3 DSC curve of betulin-TPC copolymer from -30 to $300{ }^{\circ} \mathrm{C}$

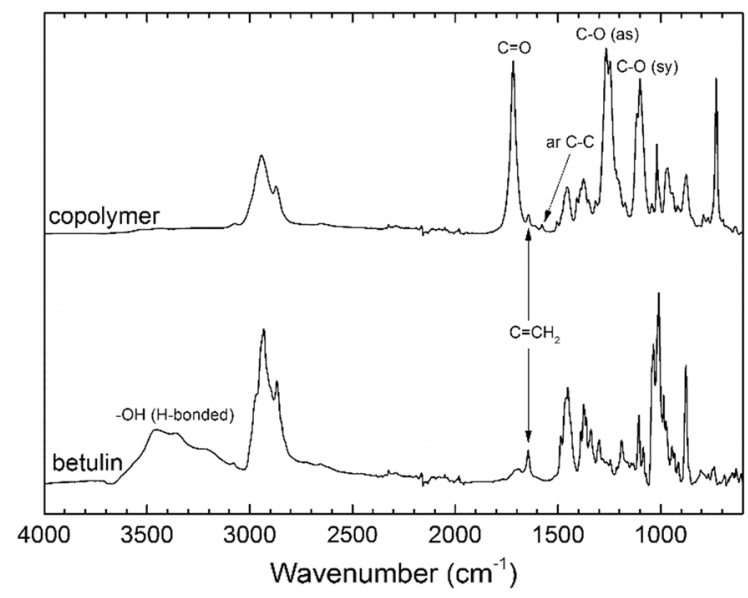

Fig. 4 FTIR spectra of betulin and betulin-TPC copolymer

betulin, the broad band of hydrogen-bonded hydroxyls was absent in the spectrum of betulin-TPC copolymer, indicating that betulin was acylated to a great extent. A weak absorption band at $1578 \mathrm{~cm}^{-1}$ due to $\mathrm{C}-\mathrm{C}$ vibration in the aromatic ring appeared in the spectrum of betulin-TPC copolymer, which could further verify the success of acylation of betulin.
Film preparation

Betulin based films with different mass ratios of cellulose triacetate to betulin are shown in Fig. 5a. Apparently, the film becomes less homogeneous when the proportion of betulin is increased, probably due to the limited solubility of betulin in chloroform. The undissolved betulin appeared as particles distributed nonuniformly on the upper surface of the film.

Betulin-TPC copolymer based films and a reference cellulose triacetate film are shown in Fig. 5b. Film 11 shows a surface with dense dots, which might be bubbles resulting from the air trapped in the viscose solution that was used to cast this film.

The water contact angle of each film was measured to determine the changes in hydrophobicity caused by the different amounts of added betulin. The reference film consisting of neat cellulose triacetate exhibited the lowest average water contact angle of approximately $80^{\circ}$ during the detection period, indicating that it was hydrophilic. Film 4 showed the highest hydrophobicity with an average water contact angle slightly lower than $125^{\circ}$, as shown in Fig. 6. The average contact angle of water on the rest of the films was between that of the reference film and film 4 . In general, the water contact angle on each film decreased only slowly, indicating that all the films possess a relatively steady wettability. Interestingly, film 1 had an initial water contact angle only slightly higher than $85^{\circ}$, indicating that this sample was unable to reach a hydrophobic level due to the limited content of betulin. It is noteworthy that the average water contact angle increased with increasing proportion of betulin in the first four samples, but for the two samples with highest betulin mass content, films 5 and 6 , the water contact angle was less than that of film 4 . This phenomenon requires further study, but a 
(a)

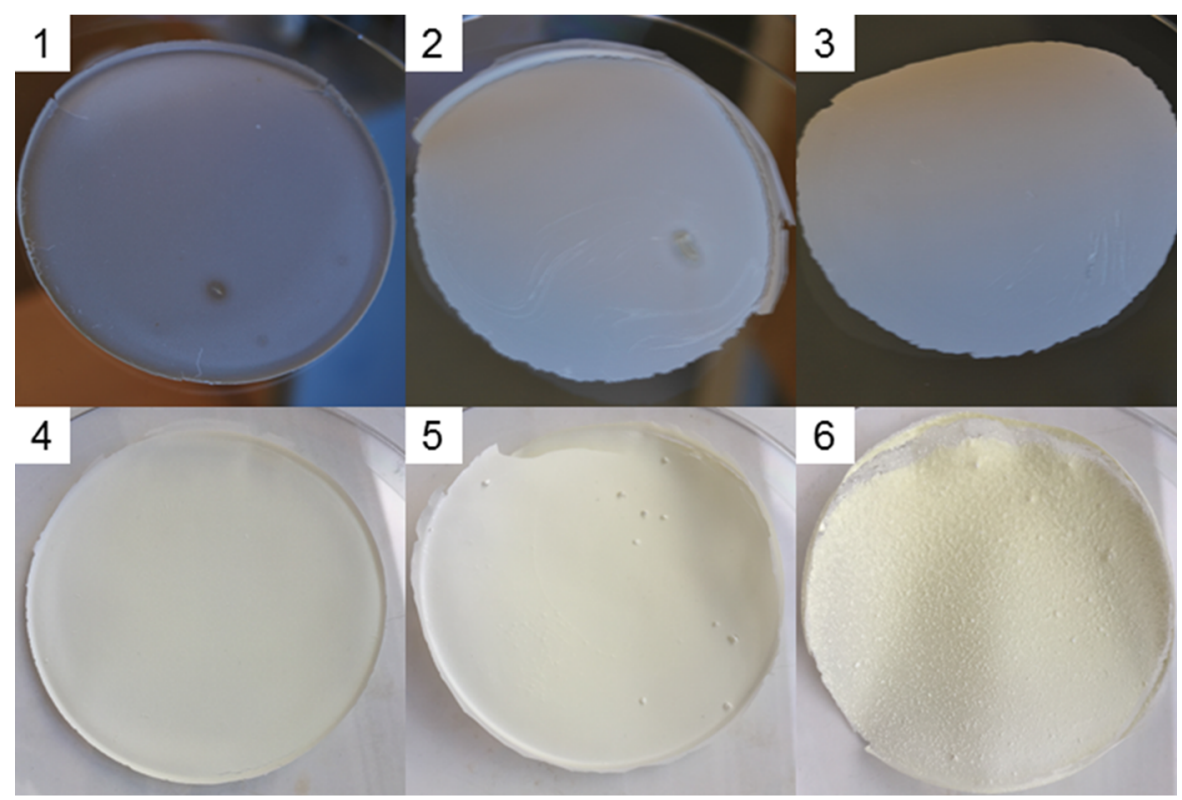

(b)

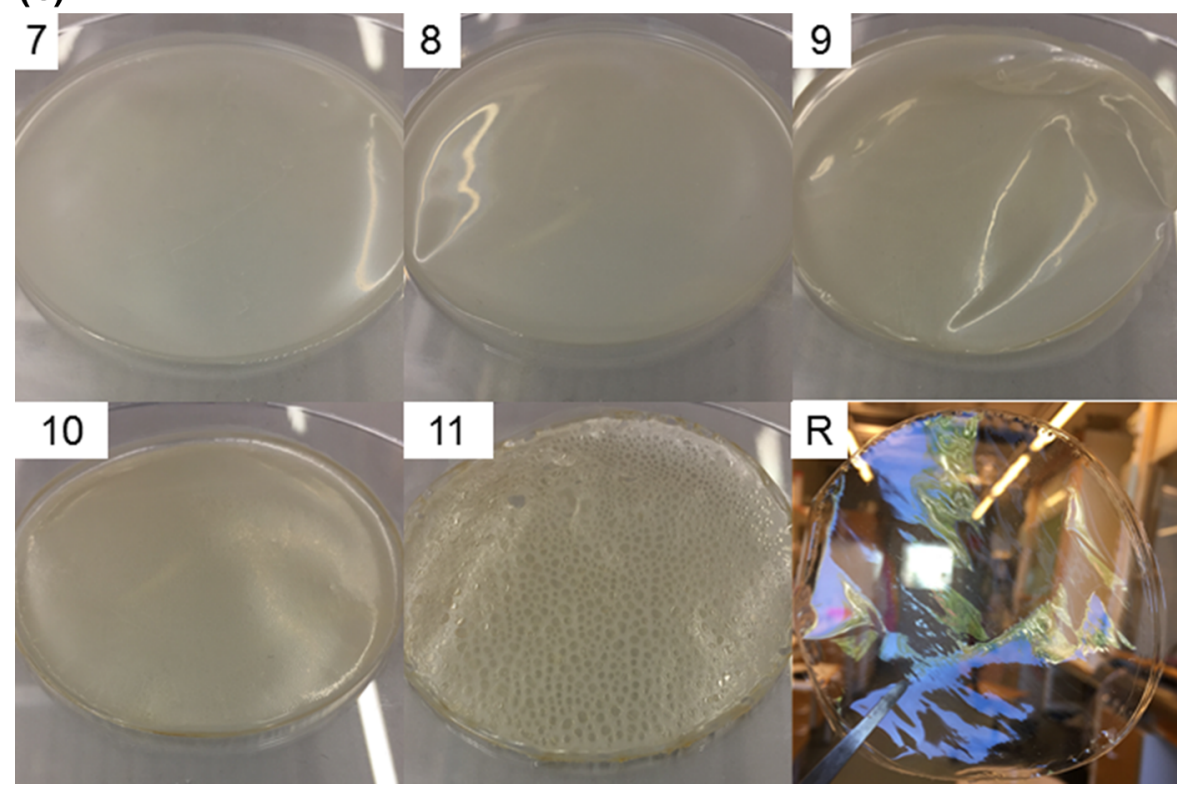

Fig. 5 a 1-6: betulin based films 1-6. b 7-11: betulin-TPC copolymer films 7-11; R: CTA reference film

hypothesis is that the distribution of betulin was inhomogeneous, as seen in Fig. 5a.

The water contact angles of films 7-11 are shown in Fig. 7. In general, for a given mass fraction, the betulin-TPC copolymer based film shows a lower water contact angle than the betulin based film, except that the initial and average contact angle of film 7 was higher than that of film 1 . Of all the 5 films, film 9 had the best general hydrophobicity with an average contact angle of approximately $100^{\circ}$. Films 4 and 9 were herein selected for coating on the cotton fabric to improve its water repellency. So far, 6 different materials were prepared with different methods, as specified in Table 2. 


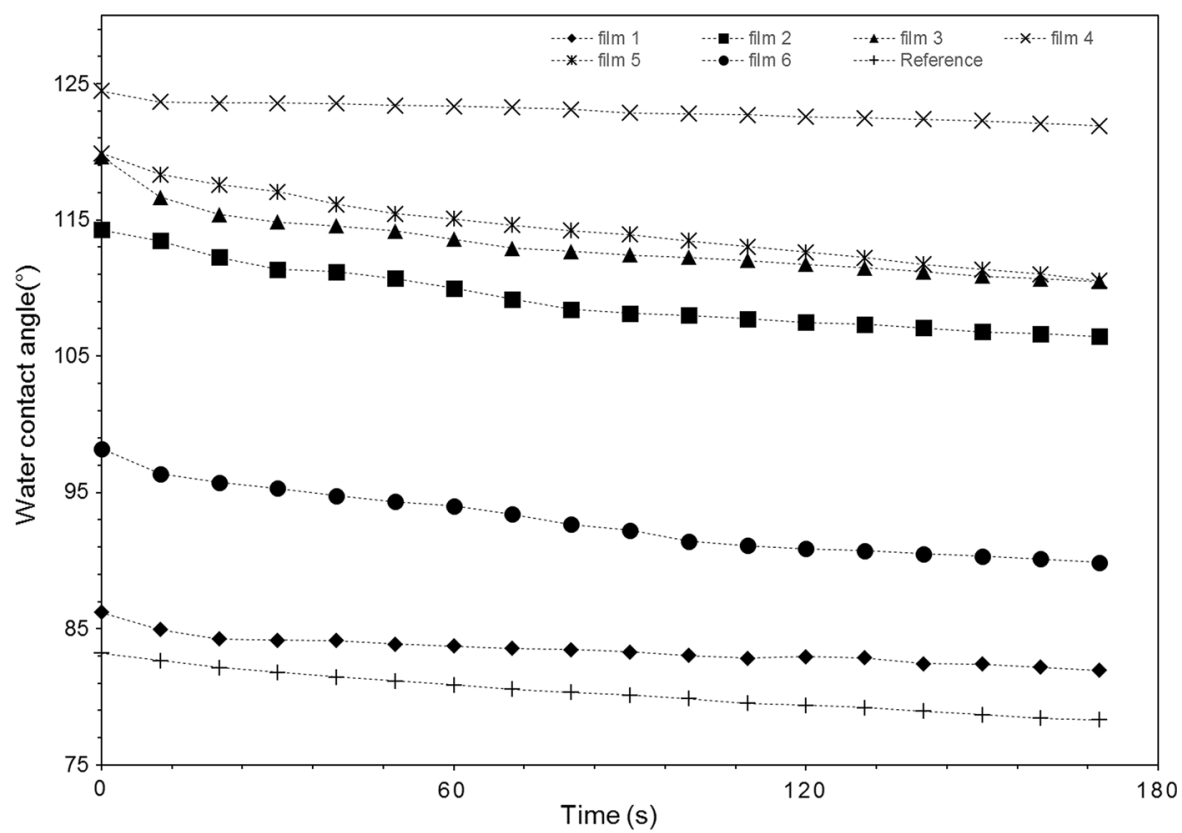

Fig. 6 Water contact angle of films 1-6 and the reference CTA film as a function of time

Fig. 7 Water contact angle of films 7-11 and a reference CTA

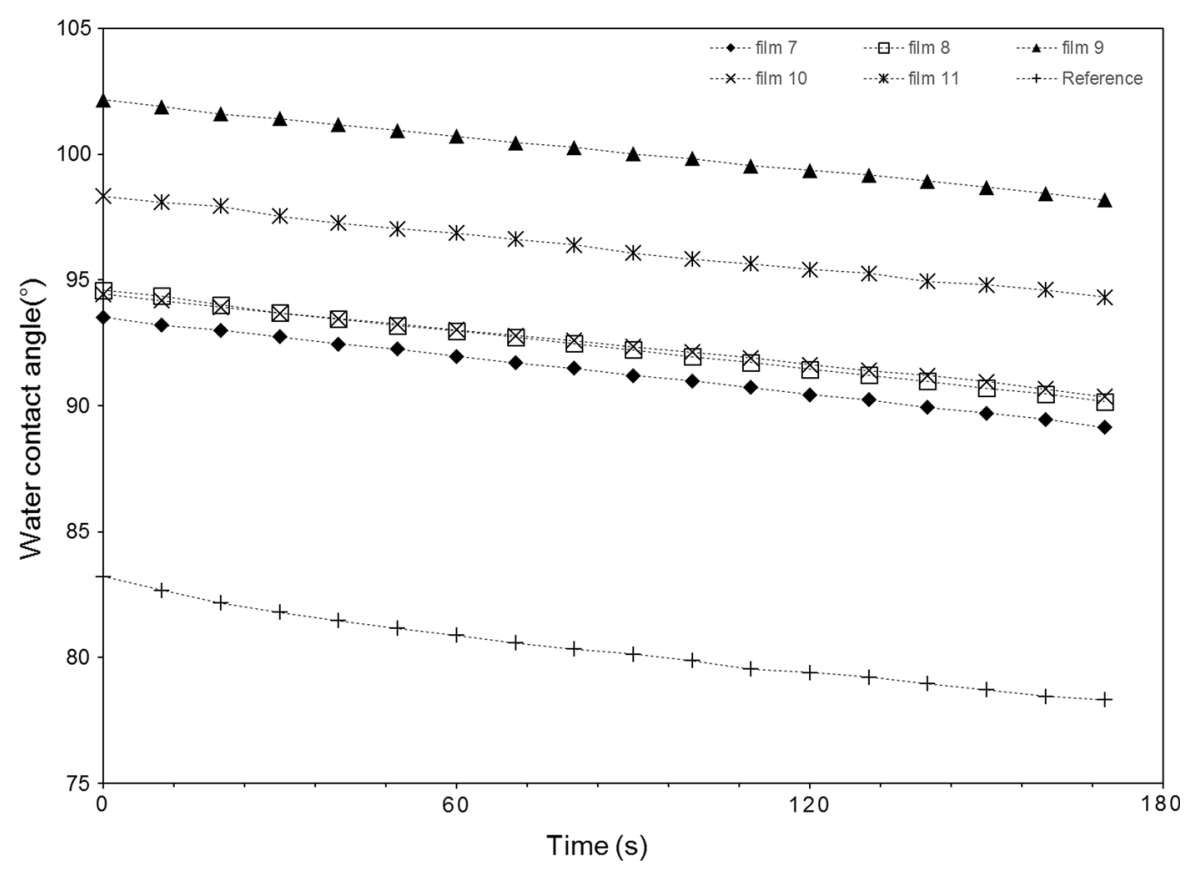

Materials preparation

\section{SEM}

Changes in the morphology of fabric fibers of materials A1, A2, A3 and B were observed by SEM
(Fig. 8). Compared with (d) the untreated sample that exhibited smooth cellulose fibers, betulin particles could be clearly observed on the fibers of samples (a) A1, (c) A2 and (e) A3. The particles were not, however, uniformly distributed. Lots of particles gathered together in some areas while other areas 
Table 2 Experimental design of materials preparation

\begin{tabular}{ll}
\hline Materials & Methods of preparation \\
\hline A1 & Fabric impregnated with $3.75 \mathrm{~g} \mathrm{~L}^{-1}$ betulin solution in ethanol \\
A2 & Fabric impregnated with $7.5 \mathrm{~g} \mathrm{~L}^{-1}$ betulin solution in ethanol \\
A3 & Fabric impregnated with $7.5 \mathrm{~g} \mathrm{~L}^{-1}$ betulin solution in ethyl acetate \\
B & Fabric impregnated with $3.75 \mathrm{~g} \mathrm{~L}^{-1}$ betulin-TPC copolymer solution in THF \\
C & Fabric coated with betulin based film (film 4, CTA: betulin $=1: 1.3)$ \\
D & Fabric coated with betulin-TPC copolymer based film (film 9, CTA: betulin-TPC copolymer $=1: 1)$ \\
\hline
\end{tabular}
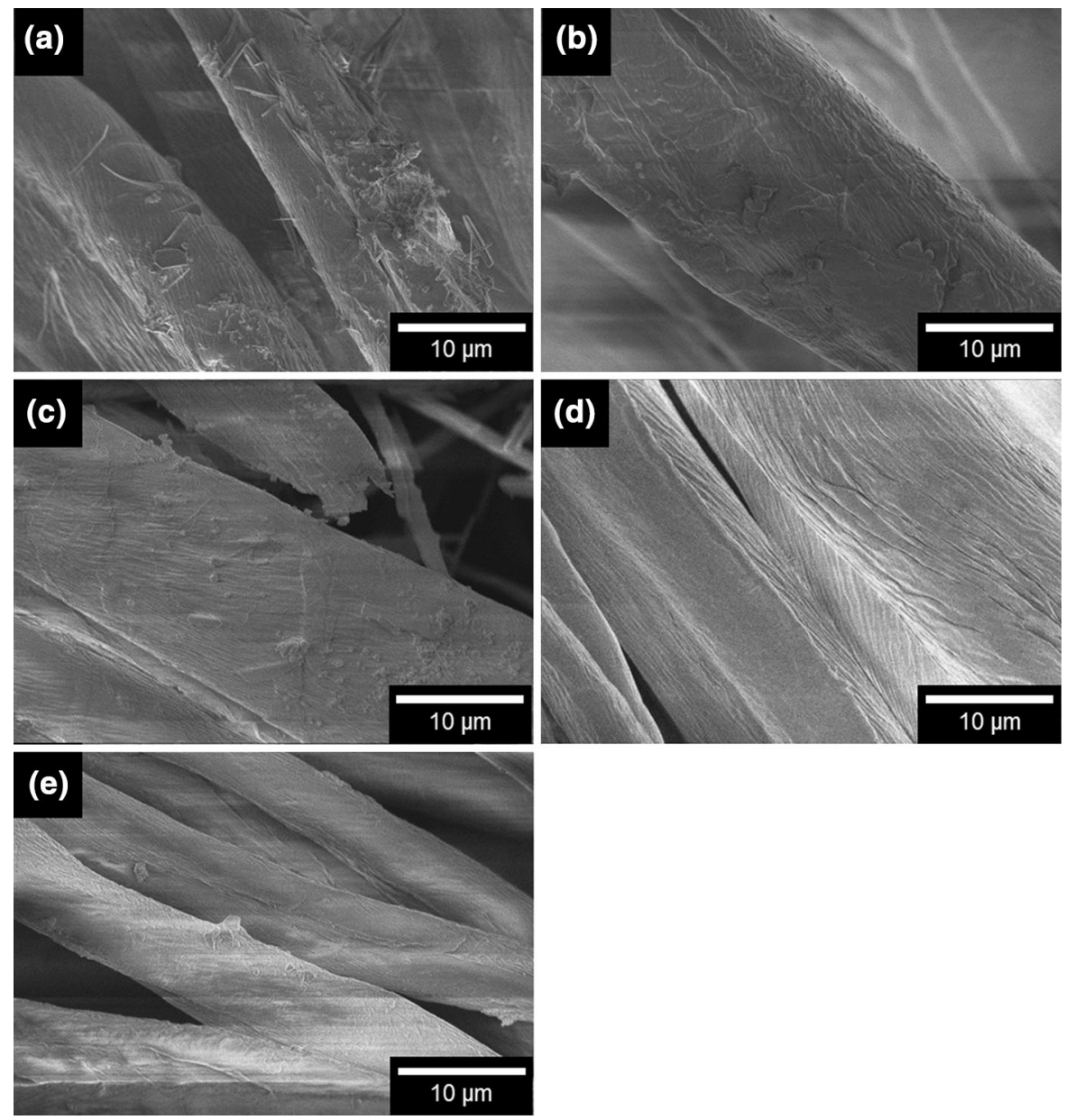

Fig. 8 SEM images of the fiber morphology of materials, a A1, c A2, e A3, b B and $\mathbf{d}$ a reference sample

were covered with only a few particles. Interestingly, some betulin particles formed thin cylinders. In the picture area, little difference in particle amount was seen in (a) A1 and (c) A2, even though the concentration of the latter is twice that of the former.
It can be seen in sample (b) B that there were pieces of copolymer with irregular shapes covering the surfaces of the fibers.

The surfaces of $(a-d)$ coated side and $(e, f)$ the fractured cross section of (a, c, e) material $\mathrm{C}$ and $(b, d$, 
f) D were also observed by SEM, Fig. 9. Figures (a) and (b) show the general morphology of materials $\mathrm{C}$ and $\mathrm{D}$ respectively at a low magnification of $30 \times$. Material C (a) presents a textured surface caused by the plain woven pattern of the fabric beneath, whereas material $\mathrm{D}$ (b) in contrast, presents a smooth surface with only a trace of crack and fragments. At a higher magnification of $400 \times$, (c) and (d), the shadow parts in (a) were revealed to be exposed textile fibers from underneath, as seen in (c). A hypothesis is that the film cracked during the compression molding process so that the textile fibers underneath were exposed. In contract, as shown in (d), material D still shows an extremely smooth surface, with an advantage over material $\mathrm{C}$. Since (d) was captured at the edge of the sample, the uncovered fabric fibers were clearly visible in the image area. The fractured cross section of both the materials are shown in (e) and (f) with a magnification of $700 \times$. As expected, for both materials, the boundary can easily be seen, indicating that the coating did not penetrate into the fabric substrate.

\section{Water contact angle}

Figure 10 shows the average contact angle as a function of time for the different materials. The variation for each series was no more than $5^{\circ}$ or less, which means that the contact angle was stable over $170 \mathrm{~s}$ for each material. A2 $\left(153^{\circ}\right), \mathrm{B}\left(151^{\circ}\right)$, A3 $\left(149^{\circ}\right)$, A1 $\left(147^{\circ}\right), \mathrm{C}\left(123^{\circ}\right), \mathrm{D}\left(104^{\circ}\right)$ could be identified as a descending sequence of average water contact angles over this time period.

Material A2 possessed a slightly higher $\left(6^{\circ}-7^{\circ}\right)$ water contact angle than $\mathrm{A} 1$, which proves that the
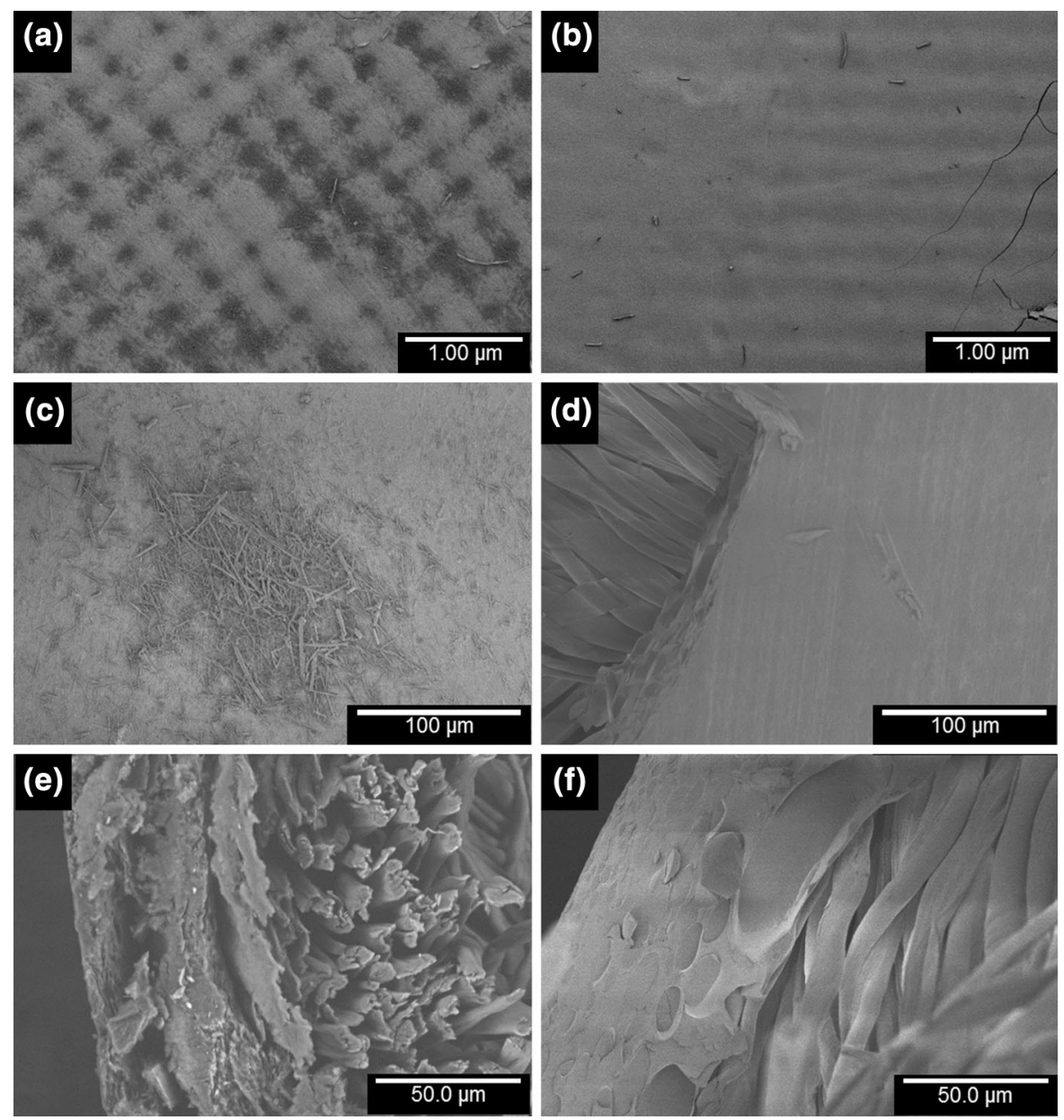

Fig. 9 SEM images of material $\mathbf{a}, \mathbf{c}, \mathbf{e} \mathrm{C}$ and $\mathbf{b}, \mathbf{d}, \mathbf{f} \mathrm{D}$. $\mathbf{a}, \mathbf{b}$ Top view with $\times 30$; $\mathbf{c}, \mathbf{d}$ top view with $\times 400$;, $\mathbf{f}$ cross section view with $\times 700$ 
Fig. 10 Water contact angle of materials A1, A2, A3, B, C and D

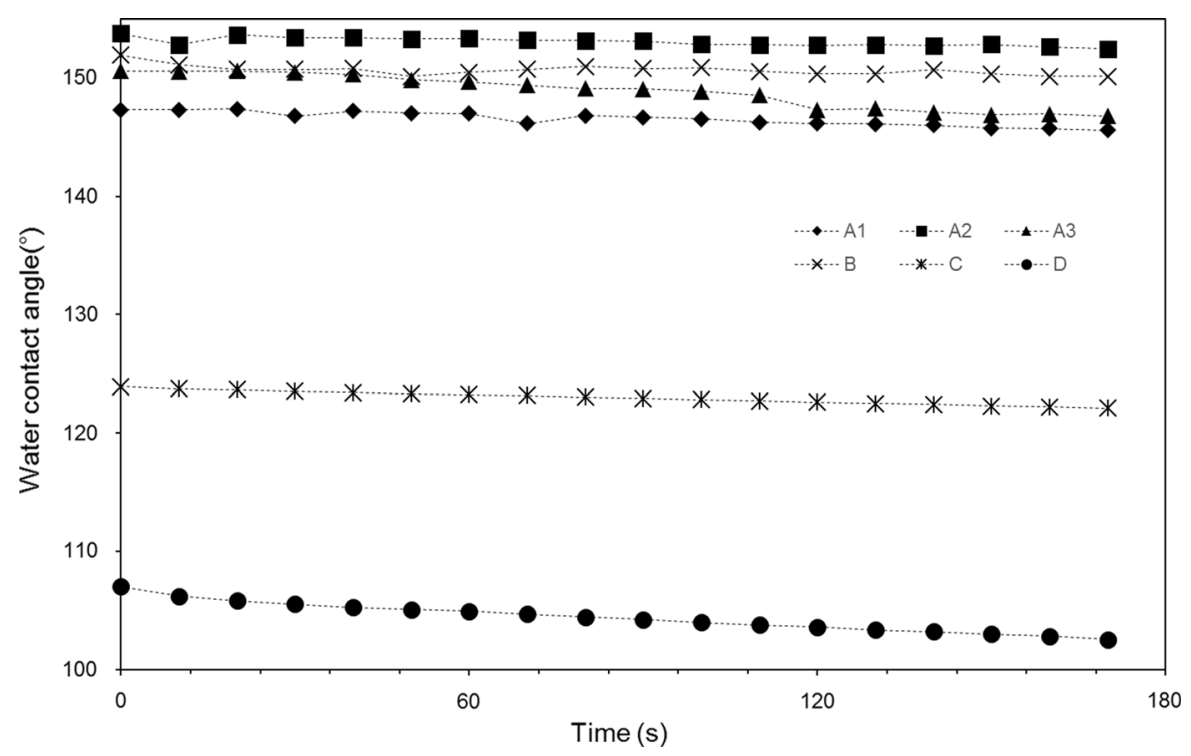

fabric treated with betulin solution in ethanol at a higher concentration has a slightly higher hydrophobicity. A difference of approximately $5^{\circ}$ was seen between $\mathrm{A} 2$ and $\mathrm{A} 3$, which shows that at the same concentration, the fabric treated with the betulin solution in ethanol exhibits a slightly higher hydrophobicity than the fabric treated with the solution in ethyl acetate. The water contact angle of the material $\mathrm{B}$ was almost stable, and the value was approximately $150^{\circ}$, which is lower than that of A2 but higher than that of the other four.

A surface with a static water contact angle higher than $150^{\circ}$ and a contact angle hysteresis less than $10^{\circ}$ can be categorized as a superhydrophobic surface (Bhushan and Jung 2008). It is noteworthy that A2 and $B$ reached the aspect of static water contact angle in the criterion of superhydrophobicity. Betulin has relative low surface energy due to its abundant hydrocarbon moieties in its structure, and the surface of cotton fabric is rough because of its woven texture formed by hairy fibers. The combination of low surface energy provided by adhered betulin and surface roughness of the fabric will generally result in a surface with high static contact angle of water, according to Cassie's law (Cassie and Baxter 1944). However, A3 did not reach the superhydrophobic level for most of the time, and A1 did not reach that for all the $170 \mathrm{~s}$, although the difference in average contact angle between samples $\mathrm{A} 2$ and $\mathrm{A} 1$ is only about $7^{\circ}$. In other words, the hydrophobicity of all these materials prepared by immersion method are more or less the same. On the other hand, the film coating method changed the wettability of the fabric samples from hydrophilic to hydrophobic, since materials $C$ and D show water contact angles higher than $90^{\circ}$, although not close to $150^{\circ}$ like the other four. This indicates that materials prepared by the immersion method have a higher hydrophobicity than those prepared by the film coating method. On the other hand, materials prepared by immersion method were washed in a washing machine at $40{ }^{\circ} \mathrm{C}$ for $2 \mathrm{~h}$ to test their durability in our recent work (Huang et al. 2016). The contact angle of materials prepared from betulin solution (material A) reduced to 0 after $20 \mathrm{~s}$, indicating that the adhered betulin particles were mostly removed; In contrast, material prepared from betulin-TPC copolymer solution (material B) showed a reduction in contact angle from $126^{\circ}$ to $70^{\circ}$ within $3 \mathrm{~min}$, indicating that material adhered with betulin-TPC copolymer has a more durable property than those adhered with betulin monomers.

\section{Water repellency}

The AATCC water repellency test is a popular method for determining the extent of water repellency achieved on fabric samples (Kale and Palaskar 2011), and this method has been used to determine the water repellency of each material in the present study, using a rating chart with different wetting 
Table 3 AATCC spray test rating standards. Reproduced with permission from AATCC TM22-2014, water repellency: spray test

\begin{tabular}{ll}
\hline Rating & Evaluation \\
\hline 100 & No sticking or wetting of the specimen face \\
90 & Slight random sticking or wetting of the specimen face \\
80 & Wetting of specimen face at spray points \\
70 & Partial wetting of the specimen face beyond the spray points \\
50 & Complete wetting of the entire specimen face beyond the spray points \\
0 & Complete wetting of the entire face of the specimen \\
\hline
\end{tabular}

pattern, as described in Table 3. Material A2 was selected as a representative of all the three materials prepared by method A, since A2 showed the best hydrophobicity of A1, A2 and A3. The results of the standard spray test for the different materials and the reference sample are shown in Fig. 11k-o. Photographs $11(\mathrm{k})$ and (l), for materials $\mathrm{A} 2$ and B respectively show that thickly dotted water droplets were presented around the central region, where the pattern is like that on the reference sample with a score of 0 . It is suggested that this is because the betulin particles in the central region were washed away by the sprayed water, while those located outside this region were not influenced. According to the standard rating chart, the scores for both materials $\mathrm{A} 2$ and $\mathrm{B}$ were approximately 70, except for the central parts, with a score of 50 or less. The durability of material A2 and B was tested in our recent work (Huang et al. 2016) and both the washed materials showed a score of 0 after laundering, indicating that the hydrophobic coating was not resistant to the laundering. Durability is an important parameter from the perspective of application and will be optimized in near future. Figures $11 \mathrm{~m}$, n, corresponding respectively to material $\mathrm{C}$ and $\mathrm{D}$, showed that the dotted water droplets were distributed uniformly, and the scores for

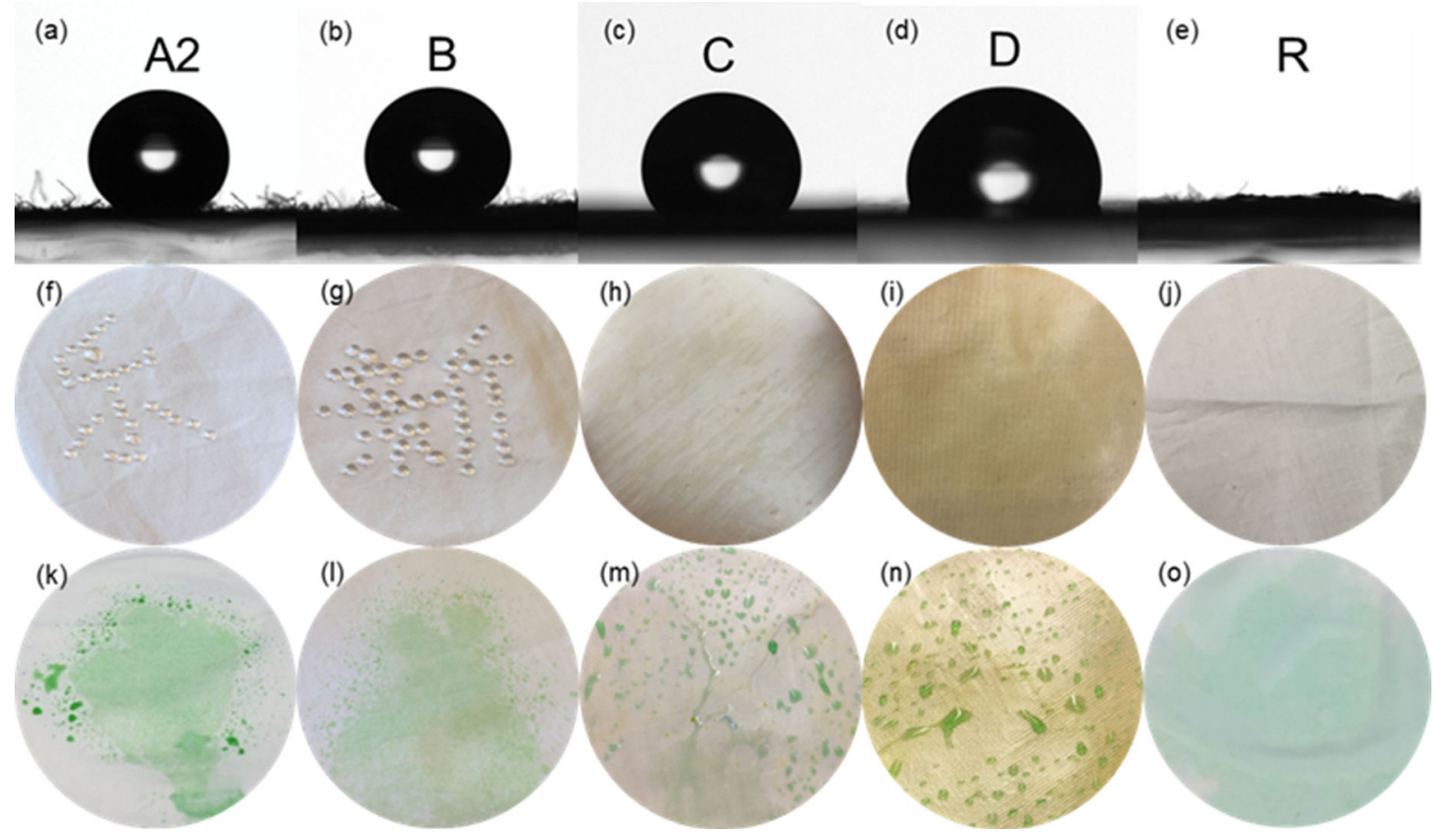

Fig. 11 Surface morphology and wetting of a, $\mathbf{f}, \mathbf{k}$ material A2, $\mathbf{b}, \mathbf{g}, \mathbf{I}$ material B, c, h, m material C, $\mathbf{d}, \mathbf{i}, \mathbf{n}$ material D and $\mathbf{e}, \mathbf{j}$, $\mathbf{o}$ an untreated reference fabric: $\mathbf{a}-\mathbf{e}$ side views of water droplets of $10 \mu \mathrm{L}$ used in water contact angle measurements; $\mathbf{f}-$ j photographs of original materials and the reference sample; $\mathbf{k}-\mathbf{o}$ photographs of materials and the reference sample after the standard spray test 
materials $\mathrm{C}$ and $\mathrm{D}$ are about 80 . The function of the covering hydrophobic films was obvious in this case since no obvious low-score regions were visible in materials $\mathrm{C}$ and $\mathrm{D}$. Although it has been shown that the water repellency can be improved by coating a hydrophobic film onto a fabric, the flexibility and air permeability of the coating need to be considered in future work.

\section{Conclusion}

In this study, betulin was isolated from the outer bark of birch through a simple solvent extraction, and a betulin-terephthaloyl chloride (TPC) copolymer was successfully synthesized. Different ways of using betulin and the copolymer for the surface-treatment of cotton fabric were explored. Cotton fabric coated with particles of betulin monomer, small pieces of the betulin-TPC copolymer, as well as films based on betulin or the copolymer showed significantly improved hydrophobicity and water repellency. All used treatments were simple and straightforward. Herein, we demonstrated that betulin, a naturally abundant and hydrophobic compound obtained from a side stream product in forest product industry, could be used for a value-added application instead of being burnt together with the bark as a solid fuel.

Acknowledgment Tianxiao Huang expressed great appreciation to the China Scholarship Council for the financial support for his study.

Open Access This article is distributed under the terms of the Creative Commons Attribution 4.0 International License (http:// creativecommons.org/licenses/by/4.0/), which permits unrestricted use, distribution, and reproduction in any medium, provided you give appropriate credit to the original author(s) and the source, provide a link to the Creative Commons license, and indicate if changes were made.

\section{References}

AATCC TM22-2014, Water repellency: spray test. https:// members.aatcc.org/store/tm22/487/. Accessed $10 \mathrm{Apr}$ 2016

Abidi N, Hequet E (2004) Cotton fabric graft copolymerization using microwave plasma. I. Universal attenuated total reflectance-FTIR study. J Appl Polym Sci 93:145-154
Bae GY, Min BG, Jeong YG, Lee SC, Jang JH, Koo GH (2009) Superhydrophobicity of cotton fabrics treated with silica nanoparticles and water-repellent agent. J Colloid Interface Sci 337:170-175

Bhushan B, Jung YC (2008) Wetting, adhesion and friction of superhydrophobic and hydrophilic leaves and fabricated micro/nanopatterned surfaces. J Phys Condens Matter 20:225010

Cassie A, Baxter S (1944) Wettability of porous surfaces. Trans Faraday Soc 40:546-551

Fridén ME et al (2016) Evaluation and analysis of environmentally sustainable methodologies for extraction of betulin from birch bark with a focus on industrial feasibility. Green Chem 18:516-523

Hagenmaier RD, Shaw PE (1991) Permeability of coatings made with emulsified polyethylene wax. J Agric Food Chem 39:1705-1708

Huang T, Li D, Ek M (2016) Hydrophobic coating on cellulosic textile material by betulin and a betulin based polymer. Dissertation, Linnaeus University. http://www.diva-portal. org/smash/get/diva2:936312/FULLTEXT01.pdf. Accessed 10 Nov 2017

Ivanova N, Zaretskaya A (2010) Simple treatment of cotton textile to impart high water repellent properties. Appl Surf Sci 257:1800-1803

Jeromenok J (2012) Polymers from the natural product betulin: a microstructural investigation. Universitätsbibliothek der Universität Potsdam, Potsdam

Kale KH, Palaskar S (2011) Atmospheric pressure plasma polymerization of hexamethyldisiloxane for imparting water repellency to cotton fabric. Text Res J 81:608-620

Krasutsky PA (2006) Birch bark research and development. Nat Prod Rep 23:919-942

Lee M, Nishi K, Jeong DS, Tokuyama T, Itazu T, Miyaji Y, Wakida T (2005) Change of surface characteristic of cotton and polyester fabrics treated with silicone resin by washing and subsequent heat treatment. Sen'i Gakkaishi 61:309-312

Mazurek M, Kinning D, Kinoshita T (2001) Novel materials based on silicone-acrylate copolymer networks. J Appl Polym Sci 80:159-180

Sahin B (1996) Fluorochemicals in textile finishing. Int Text Bull Dye Print Finish 42:26-30

Sharoyan DE (2011) Pulping additives for a reduction of resin from Kraft pulp. U.S. Patent No. 8,052,840

Shi Z, Wyman I, Liu G, Hu H, Zou H, Hu J (2013) Preparation of water-repellent cotton fabrics from fluorinated diblock copolymers and evaluation of their durability. Polymer 54:6406-6414

Singh O (1987) Stain removal characteristics of fabrics and stain resistance/release finishing: a review. Text Dyer Print 20:24-27

Vasnev VV, Konkina IN, Korshak VV, Vinogradova SV, Lindberg JJ, Jääskeläinen P, Piiroinen K (1987) Syntheses of unsaturated polyesters containing betulinol moieties. Macromol Chem Phys 188:683-691

Wang Q, Fan X, Gao W, Chen J (2006) Characterization of bioscoured cotton fabrics using FT-IR ATR spectroscopy and microscopy techniques. Carbohyd Res 341:2170-2175

Wenzel RN (1936) Resistance of solid surfaces to wetting by water. Ind Eng Chem 28:988-994 
Wi D-Y, Kim IW, Kim J (2009) Water repellent cotton fabrics prepared by PTFE RF sputtering. Fibers Polym 10:98-101

Xu B, Cai Z (2008) Fabrication of a superhydrophobic ZnO nanorod array film on cotton fabrics via a wet chemical route and hydrophobic modification. Appl Surf Sci 254:5899-5904
Yahaya G, Brisdon B, Maxwell M, England R (2001) Preparation and properties of functionalized polyorganosiloxanes. J Appl Polym Sci 82:808-817

Young T (1805) An essay on the cohesion of fluids. Philos Trans R Soc Lond 95:65-87 